\title{
Erratum to: MHC evolution in three salmonid species: a comparison between class II alpha and beta genes
}

\author{
Daniela Gómez • Pablo Conejeros • Sergio H. Marshall • \\ Sofia Consuegra
}

Published online: 1 April 2011

(C) Springer-Verlag 2011

Erratum to: Immunogenetics (2010) 62 531-542

DOI: $10.1007 / \mathbf{s 0 0 2 5 1 - 0 1 0 - 0 4 5 6 - x}$

In Table 2 the sequence of the primers read:

O. kisutch and O. mykiss DAB Forward: AGGACCTG CATGgtatAgAg Reverse: CTTGTCCAGTATGGC GCTGATG

The correct sequence is:

O. kisutch and O. mykiss DAB Forward: GTGTCGA TACTCCTCAAAGGAC Reverse: CTTGTCCAGTATGG CGCTGTAG

The online version of the original article can be found at http://dx.doi. org/10.1007/s00251-010-0456-x

D. Gómez $\cdot$ P. Conejeros $\cdot$ S. H. Marshall

Laboratorio de Genética e Inmunología Molecular,

Instituto de Biología, Facultad de Ciencias Básicas y

Matemáticas, Pontificia Universidad Católica de Valparaíso,

Valparaíso, Chile

S. Consuegra $(\bowtie)$

Institute of Biological, Environmental and Rural Sciences,

Aberystwyth University,

Edward Llwyd Building Penglais Campus,

Ceredigion SY23 3DA Wales, UK

e-mail: skc@aber.ac.uk 